\title{
LEVELS OF REPRODUCTIVE HORMONES IN TREATMENT-NAÏVE HYPERTENSIVE VOLUNTEERS IN LAGOS
}

\author{
*Ekun, O. A., Ossai, B. C., Ogidi, O. N. and Odia, O. A. \\ Department of Medical Laboratory Science, College of Medicine of the University of Lagos. \\ *Corresponding Author: E-mail: ayodele1619.oe@gmail.com +2348033151619 \\ (Received: $5^{\text {th }}$ October, 2017; Accepted: $29^{\text {th }}$ November, 2017)
}

\section{ABSTRACT}

\begin{abstract}
High blood pressure is a medical condition characterized with persistent elevated systolic and or diastolic blood pressure. It usually presents no symptom in most cases and if untreated it eventually leads to complications. Thus the aim of this study is to evaluate the possible effect of high blood pressure on male reproductive hormones level. A total number of one hundred and ten male volunteers between ages 18-55years participated in this study. Out of this, sixty were hypertensive without previous knowledge of this as at the time of this study hence were not on antihypertensive medication. The hypertensive volunteers served as test group, they were identified through routine check. The remaining fifty served as control group. The anthropometric measurement was carried out on all volunteers. Five millimeters of blood sample was collected from each patient and was assayed for testosterone, luteinizing hormone ( $\mathrm{LH}$ ), follicle stimulating hormone (FSH) using Enzyme Linked Immunosorbent assay (ELISA). There was a significant increase $(\mathrm{p}<0.05)$ in the mean systolic, diastolic blood pressure, body mass index and waist circumference among treatment naïve hypertensive group. On the other hand, the mean total testosterone value among the test group was significantly lower $(\mathrm{p}<0.05)$ than the control group while the mean follicle stimulating hormone (FSH) value was significantly higher among the test group $(\mathrm{p}<0.05)$ when compared with control. There was a negative but insignificant $(p>0.05)$ association between age and testosterone, age and luteinizing hormone, follicle stimulating hormone and testosterone, body mass index and testosterone. Based on these findings, this study opined that high blood pressure may disrupt the normal reproductive hormones homeostasis in male and should not be taken for granted.
\end{abstract}

Keywords: Hypertension, Male, Reproduction hormones and Anthropometric study.

\section{INTRODUCTION}

High blood pressure is a chronic medical condition in which the blood pressure in the arteries is persistently elevated. It is also known as arterial hypertension (James et al., 2014). Systolic and diastolic pressures are used to express blood pressure measurements which are maximum and minimum pressures respectively. Systolic pressure occurs when the left ventricle is most contracted, while the diastolic pressure occurs when the left ventricle is most relaxed prior to the next contraction. Normal blood pressure at rest is within the range of $90-140 \mathrm{mmHg}$ systolic and 60$90 \mathrm{mmHg}$ diastolic. High blood pressure is present if the resting blood pressure is persistently at or above 140/90 $\mathrm{mmHg}$ for most adults (James et. al.; 2014). High blood pressure usually does not cause symptoms initially but sustained hypertension over time is a major risk factor for diseases (Lewington et al., 2002).

Previous studies have evaluated the potential debilitating and devastating effects of high blood pressure on body systems with particular reference to its effects on the central nervous system leading to stroke (Sutter, 2011), chronic kidney failure (CKF), chronic heart failure (Jamerson and Agoda, 2012, Damasceno et al., 2012), and retinopathy. It is however surprising that there is a limited published information on the possible effects of high blood pressure on the reproductive health and males' fertility. The limited available data from Bhasin et al., (2008) however reported that high blood pressure possibly affects reproductive hormones' functions which elicit negative impact on male reproduction.

Hormones play a major role in the interaction of the gametes which are involved in human reproduction. The entire male reproductive system is dependent on hormones which are chemicals that stimulate or regulate the activity of cells or organs of reproduction. The primary hormones involved in male reproductive system are: follicle stimulating hormone (FSH), 
luteinizing hormone $(\mathrm{LH})$ and testosterone. It has been suggested that adult human sexual reproductive activities are affected by several pathological conditions and non-communicable metabolic disturbances. While many studies have been reported on effect of immunological disturbances on reproduction in humans, there is a paucity of information on the possible effect of high blood pressure on the chemical messengers (hormones) that regulate reproduction activities. Hence this study intends to evaluate the effect of high blood pressure on male reproductive hormones.

\section{MATERIALS AND METHODS}

\section{Volunteers' Selection and Study Design}

This study was a randomised cross sectional study conducted on male marketers who have neither been diagnosed of high blood pressure before nor been on any anti-hypertensive treatment before the time of this study. Volunteers were recruited from one major market in each of the twenty local government areas of Lagos state. The hypertensive group comprises of males with equivocal high blood pressure measurements from routine medical check conducted on consenting men volunteers. Informed consent was taken from the volunteers and a total of one hundred and ten (110) volunteers participated in this study out of which sixty were newly diagnosed hypertensive while fifty non-hypertensive participants served as control.

\section{Ethical Consideration}

Approval was obtained from the Research and Ethics Committees of Lagos University Teaching Hospital (LUTH) and College of Medicine of University of Lagos (CMUL) prior to the commencement of the study. Informed consent was sought from each of the volunteers of the study prior to participating in this study.

\section{Methodology}

General Principle of Gonadotropins ELISA Assay

Enzyme linked immuno-sorbent assay technique was used to determine the hormonal levels of participants. Samples and standards were added to a 96-well plate precoated with anti-hormone antibodies, the hormone in the sample and standards bind to the precoated antibody. After incubation and washing, anti-hormone horseradish peroxidase conjugate was added to the antibody-hormone complex and incubated, the wells were washed after incubation to remove unbound material. Tetra methyl benzidine substrate was added and allowed to react with horseradish peroxidase to produce blue coloration. The reaction was terminated with addition of stop solution containing $0.5 \mathrm{M}$ sulfuric acid which stops the color reaction and produces a color change from blue to yellow. The intensity of color is directly proportional to the amount of hormone in the sample and the intensity was measured at appropriate wavelength e.g $450 \mathrm{~nm}$. The absorbance for each calibrator was plotted against their corresponding concentration in $\mathrm{mIU} / \mathrm{L}$ or IU/L on linear graph paper. The bestfit curve was drawn through the plotted points.

The concentration of the hormones for the unknown samples was determined by locating the absorbance for each unknown samples on the vertical axis of the graph, finding the intersecting point on the curve, and the concentration from the horizontal axis of the graph.

\section{Protocol for Follicle Stimulating Hormone Assay}

$50 \mu \mathrm{l}$ standards, control and samples were pipetted into their respective wells, the first well was left empty. This served as a blank. $100 \mu$ l of enzyme conjugate of horseradish peroxidase was added into each well. It was mixed gently for 30 seconds. The wells were covered with the foil supplied with the kit and incubated at room temperature for 60 minutes. The content of each well was aspirated and the wells were washed five times with washing solution. After washing the plate was inverted and blotted against clean paper towels to remove excess liquid. $100 \mu \mathrm{l}$ tetra methyl benzidine reagent was added into each well and mixed for 10 seconds. The plate was then incubated at room temperature for 15 minutes in the dark. The reaction was stopped with addition of $50 \mu$ l of stop solution containing $0.5 \mathrm{M}$ sulfuric acid to each well and mixed gently for 30 seconds. The absorbance of the sample was measured with micro plate reader at $450 \mathrm{~nm}$ within 30 minutes of addition of stop solution.

\section{Protocol for Luteinizing Hormone Assay}


$50 \mu 1$ standards, control and samples were pipetted into their respective wells. $100 \mu \mathrm{l}$ luteinizing hormone horseradish peroxidase and biotin conjugate was added to each well. A blank well was left for substrate blank. The wells were covered with the foil supplied with the kit and incubated for 1 hour at room temperature. It was washed with wash buffer and blotted against clean paper towels to remove excess liquid. $100 \mu l$ tetra methyl benzidine substrate solution was added into all wells. It was incubated for exactly 15 minutes at room temperature in the dark. $50 \mu \mathrm{l}$ stop solution containing $0.5 \mathrm{M}$ sulfuric acid was added into all wells in the same order and solution mixed gently. The absorbance of the sample was measured at $450 \mathrm{~nm}$ within 30 minutes of addition of stop solution.

\section{Protocol for Testosterone Assay}

$25 \mu \mathrm{l}$ calibrators, control and samples were pipetted into their respective wells. $100 \mu \mathrm{l}$ testosterone-HRP conjugate was added to each well. The wells were covered with the foil supplied with the kit and incubated for 1 hour at $37^{\circ} \mathrm{C}$. It was washed with wash buffer and blotted against clean paper towels to remove excess liquid $100 \mu$ l TMB substrate solution was added into all wells. It was incubated for exactly 15 minutes at room temperature in the dark. $100 \mu \mathrm{l}$ stop solution was added into all wells in the same order and mixed gently. The absorbance of the sample was measured at $450 \mathrm{~nm}$ within 5 minutes of addition of stop solution.

\section{Data Analysis}

The data collected from the study were analyzed using SPSS version 21.0. Quantitative data was expressed as mean \pm standard error of mean. Independent student " $t$ " test was used to compare the groups. Pearson correlation coefficient determination was performed to evaluate the degree of association. Probability value of less than $0.05 \quad(p<0.05)$ was considered to be statistically significant.

\section{RESULTS AND DISCUSSION}

Table 1 shows the mean anthropometric parameters, systolic and diastolic blood pressure. There was a significant increase in the systolic and diastolic blood pressure $(p<0.000)$ among test group when compared to the control group. The mean age of the test group and control were not significantly different from each other $(p=0.446)$. On the other hand, the mean waist circumference and the body mass index (BMI) were significantly $(p=0.011)$ higher in test group, while the test group were observed to be significantly shorter than the control $(\mathrm{p}=0.018)$.

Table 1: The Mean \pm SEM of Anthropometric Data for the Test and Control Volunteers

\begin{tabular}{lrrrl}
\hline VARIABLES & \multicolumn{1}{c}{$\begin{array}{c}\text { TEST } \\
\text { N=60 }\end{array}$} & $\begin{array}{c}\text { CONTROL } \\
\mathrm{N}=50\end{array}$ & t-test & p-value \\
& & & & \\
AGE (years) & $47.28 \pm 0.76$ & $48.07 \pm 2.06$ & 0.776 & 0.446 \\
HEIGHT (m) & $5.57 \pm 0.05$ & $5.76 \pm 0.04$ & 2.45 & $0.018^{*}$ \\
WEIGHT (Kg) & $78 \pm 2.38$ & $73.7 \pm 1.81$ & 1.57 & 0.122 \\
WAIST(inches) & $38.22 \pm 0.59$ & $35.06 \pm 0.80$ & 2.65 & $0.011^{*}$ \\
& & & & \\
BMI (Kg/m ${ }^{2}$ ) & $26.61 \pm 0.69$ & $24.99 \pm 0.60$ & 2.65 & $0.011^{*}$ \\
SYSTOLIC BP (mmHg) & $163.72 \pm 2.31$ & $126.72 \pm 1.44$ & 14.20 & $0.000^{*}$ \\
DIASTOLIC BP (mmHg) & 98.60 & $82.5 \pm 0.90$ & 8.30 & $0.000^{*}$ \\
\hline
\end{tabular}

${ }^{*} \mathrm{p}<0.05$

Furthermore, in table 2, the mean reproductive hormones level among the hypertensive and the normotensive control groups were presented. It was observed that the mean follicle stimulating hormone value among the hypertensive group was significantly higher $(p=0.001)$ than the normotensive control group; while the mean testosterone in the test group on the other hand was significantly lower $(\mathrm{p}=0.001)$ than the normotensive control group. There was no 
significant difference $(p=0.265)$ in the mean luteinizing hormone of the hypertensive and normotensive control group. A significantly $(p<0.05)$ lower mean testosterone value among test group as observed in this study is in consonance with the previous studies of Hughes et al., (1989), Jaffe et al., (1996), Dubey et al., (2002) and Fogari et al., (2005) where total serum testosterone and the bioavailable testosterone were found to be significantly lowered $(p<0.05)$ among the hypertensive volunteers when compared with the normotensive volunteers. While there are no direct and clear explanations for this, Dubey et al., (2002) however reported that lower mean testosterone values as observed among hypertensive volunteers reflects increase stress associated with hypertension. Thus it appears that hypertensive test group probably experienced mental stress which might have possibly suppress the hypothalamic-pituitarygonadal axis leading to decrease testosterone secretion (Ishikura et al., 2008). This could in a way lead to disruption of this reproductive hormone homeostasis leading to more deleterious consequences. It has been shown however that increase in body mass index (a pointer to an overweight and obesity) among hypertensive individuals could impact negatively on plasma level of testosterone as well as bioavailable testosterone; as previous study has shown that an inverse relationship exist between serum total testosterone value and body mass index (BMI) among men with hypertension and erectile dysfunction (Diaz-Arjonilla et al., 2009). Our Pearson's correlation study also clearly demonstrated (Table 3) an inverse relationship between serum total testosterone and body mass index (BMI). Thus it appears that the Pearson's correlation between testosterone and body mass index as observed in this study agreed with the previous study of Diaz-Arjonilla et al., (2009). An inverse relationship $(\mathrm{p}<0.05)$ between the mean waist circumference and testosterone value among the group studied was also observed.

Table 2: The Mean \pm SEM of the Level of Reproductive Hormones in Test and Control Volunteers

\begin{tabular}{lclcl}
\hline VARIABLE & TEST & CONTROL & t-test & p-value \\
& $\mathrm{N}=60$ & $\mathrm{~N}=50$ & & \\
\hline LH $(\mathrm{IU} / \mathrm{L})$ & $6.24 \pm 0.51$ & $5.63 \pm 0.22$ & 1.13 & 0.265 \\
FSH $(\mathrm{IU} / \mathrm{L})$ & $9.17 \pm 0.85$ & $5.32 \pm 0.47$ & 3.70 & $0.001^{*}$ \\
TESTOSTERONE $(\mathrm{ng} / \mathrm{ml})$ & $2.69 \pm 0.07$ & $3.08 \pm 0.01$ & -3.42 & $0.001^{*}$ \\
\hline
\end{tabular}
${ }^{\mathrm{p}}<0.05$

Table 3: Pearson's Degree of Association between Parameters Studied

\begin{tabular}{ccccccccc}
\hline \multicolumn{6}{c}{ Age \& wt, wt \& BMI, Age \& testo, wt \&testo, BMI \& testo, FSH\&LH, wt\&BMI, Waist\&BMI, Ht\&Waist } \\
\hline r: 0.099 & 0.833 & -0.092 & -0.359 & -0.276 & 0.444 & 0.833 & 0.778 & 0.321 \\
p: 0.451 & $0.000^{*}$ & 0.486 & $0.005^{*}$ & $0.033^{*}$ & $0.000^{*}$ & $0.000^{*}$ & $0.000^{*}$ & $0.012^{*}$ \\
\hline
\end{tabular}

$\mathrm{wt}=$ weight, $\mathrm{BMI}=$ Body mass index, Testo $=$ Testosterone, $\mathrm{FSH}=$ Follicle stimulating hormone $\mathrm{Ht}=$ Height, ${ }_{\mathrm{p}} \mathrm{p}<0.05, \mathrm{r}=$ correlation coefficient, $\mathrm{p}=$ level of probability.

In addition to this, the study of Laughlin et al., (2008) and Having et al., (2010) showed that there are clear association between low serum testosterone levels and various cardiovascular diseases such as arterial stiffness which is a common finding in hypertension (Chung et al., 2014), suggesting the possible consequence of hypertension in males probably leading to a possible subclinical hypogonadism; as subclinical hypogonadism is associated with an almost 10- fold increase risk of cardiovascular mortality, comparable to that for overt hypogonadism (Corona et al., 2006, Coronal et al., 2013). It must be noted that a significantly low total testosterone value as was observed among the hypertensive group may be an indicator to suggesting a subclinical hypogonadism.

Furthermore, it was observed that the mean LH value in test group though marginally higher than 
the control was statistically insignificant ( $p>0.05)$. This observation agrees with the previous studies of Hughes et al., (1989) and Jaffe et al., (1996). However the data from our study varied slightly from that of Jaffe et al., (1996) on the mean FSH value as we observed a significant increase in FSH value of hypertensive volunteers when compared to the normotensive control group in our study. The possible reasons for this finding are not known at this moment. This finding could possibly be an indicator supporting a gradual trend towards hypogonadism as indicated by low testosterone.

Also, we evaluated the level of association between the parameters measured among hypertensive volunteers using Pearson's correlation analysis as shown in table 3 . In this table, a negative but insignificant association was observed between volunteer's age and mean testosterone value $(p=0.486)$, age and $\mathrm{LH}$ $(p=0.440)$ respectively. On the other hand, there was a positive association between weight and waist $(\mathrm{p}=0.000)$, weight and BMI $(\mathrm{p}=0.000)$, waist and BMI $(\mathrm{p}=0.000)$ as well as FSH and LH $(\mathrm{p}=0.000)$.

A positive and significant association between $\mathrm{LH}$ and FSH as observed from Pearson's correlation analysis suggest that the two hormones are possibly influenced by similar stimulus or stimuli. It was also observed from this study that there is no clear cut direct connection or dependency between age and waist circumference as well as body mass index.

\section{CONCLUSION}

From this study, it could be inferred that hypertension in males coupled with increased body mass index and waist circumference could possibly cause a disruption in reproductive hormone homeostasis hence its management should be taken seriously in order to improve the quality of reproductive life of men with high blood pressure.

\section{REFERENCES}

Bhasin, S., Cunningham, G. R., Hayes, F.J, Matsumoto, A.M., Snyder, P.J., Swerdloff, R.S., Montori, V.M., 2006. Testosterone therapy in adult men with androgen deficiency syndromes: An Endocrine Society Clinical Practice Guideline. J Clin EndocrinolMetab. 91:1995-2010.

Bombelli, M., Facchetti, R., Sega, R., Carugo, S., Fodri, D., Brambilla, G., Giannattasio, C., Grassi, G., Mancia, G., 2011. Impact of body mass index and waist circumference on the long term risk of diabetes mellitus, hypertension, and cardiac organ damage. Hypertension. 58(6):991-3

Chung, C., Chen, H., Chang, J., Lin, Y., Hsiao, J., Chang, S., Hsu, J., 2014. Relationship between resistant hypertension and arterial stiffness assessed by brachial-ankle pulse wave velocity in the older patient. Clin Interv Aging. 9:1495-1502.

Corona, G., Mannucci, E., Petrone, L., Ricca, V., Balercia, G., Mansani, R., Chiarini, V., Giommi, R., Forti, G., Maggi, M., 2006. Association of hypogonadism and type II diabetes in men attending an outpatient erectile dysfunction clinic. Int J Impot Res. 18:190-7.

Corona, G., Rastrell, G., Monami, M., Maseroli, E., Jannini, E.A., Balercia, G., Sforza, A., Forti, G., Mannucci, E., Maggi, M., 2013. Frequency of sexual activity and cardiovascular risk in subjects with erectile dysfunction: cross-sectional and longitudinal analyses. Andrology. 1: 864-71.

Damasceno, A., Mayosi, B. M., Sani, M., Ogah, O. S., Mondo, C., Ojji. O., Dzudie, A., Maru, F., Alemayehu, B., Edwards, C., Davison, B. A., Cotter, G., Silwak., K., 2012. The Causes, treatment, and outcome of acute heart failure in 1006 Africans from a countries. Results of sub-Saharan African survey of heart failure. Arch Inter med, 172:1386-1394

Diaz-Arjonilla, M., Schwarcz, M., Swerdloff, R. S., Wang, C., 2009. Obesity, low testosterone levels and erectile dysfunction. Int J Impot Res. 21:89-98.

Dubey, R. K., James, S., Imthurn, B., Jackson, E. K., 2002. Sex hormones and hypertension. Hypertension Cardiovasc Res.53:688-708.

Fogari, R., Preti, P., Zoppi, A., Fogari, E., Rinaldi, A., Corradi, L., Mugellini, A., 2005. Serum testosterone level and arterial blood pressure in the elderly. Hypertension 
Research. 28:625-630.

Having, R. H., Steveling, A., 2010. Low serum testosterone levels are associated with increased risk of mortality in a population-based cohort of men aged 2079. Eur heart J.31:1494-1501

Hughes, G. S., Mathur, R. S., Margolius, H. S., 1989. Sex steroid hormones are altered in essential hypertension. J Hypertens. 7:181187.

Jaffe, A., Chen, Y., Kisch, E.S., Fischel, B., Alon, M., Stern, N., 1996. Erectile Dysfunction in Hypertensive Subjects Assessment of Potential Determinants. Hypertension. 28:859-862.

Jamerson, K.A., Agoda, L., 2012. Hypertension as an emerging risk factor for acute heart failure in Africa. Arch Inter med. 172:13951396.

James, P. A., James., S., Carter, B. L., Cushman, W. C., Dennison-Himmelfarb, C., Handler, J., Lackland, D. T., LeFevre, M. L., Macknzie, T. D., Ogedegbe, O., Smith, S.C. Jr., Svetkey, L. P., Taler, S. J., Townsend, R. R., Wright, J. T. Jr., Narva, A.S., Oritz, E., 2014. Evidence-Based Guideline for the Management of High Blood Pressure in Adults: Report From the Panel Members Appointed to the Eight Joint National Committee (JNC 8). JAMA 311 (5): 507-20.
Ishikura, F., Asanuma, T., Beppu, S., 2008. Low testosterone levels in patients with mild Hypertension Recovered after Antidepressant Therapy in Male Climacterium Clinic. Hypertension Research. 31:243-248

Laughlin, G.A., Barrett-Connor, E., Besrgstrem, J., 2000. Low-serum testosterone and mortality in older men. J Clin Endocrinol Metab. 93:68-75.

Lewington, S., Clarke, R., Qizilbash, N., Peto, R., Collins, R., 2002. Prospective Studies, Collaboration. "Age-specific relevance of usual blood pressure to vascular mortality: a meta-analysis of individual data for one million adults in 61 prospective studies.". Lancet 360 (9349):190.

Roka, R., 2015. Association between hypertension and body mass index and waist circumference in U.S Adults: A comparative analysis by gender: high blood press \& cardiovasc. 78:654-670.

Sutter, M., 2011. Systemic hypertension, in current medical Diagnosis and treatment method by Stephen J. Mephie and Maxine A Papadskis Chapt 11:pg 416-445. Mc Graw Hill Lange. 